\title{
Orientation Adaptive Minimal Learning Machine for Directions of Atomic Forces
}

\author{
Antti Pihlajamäki ${ }^{1}$, Joakim Linja ${ }^{2}$, Joonas Hämäläinen ${ }^{2}$, Sami Malola ${ }^{1}$, \\ Paavo Nieminen ${ }^{2}$, Tommi Kärkkäinen ${ }^{2}$ and Hannu Häkkinen ${ }^{1,3}$ * \\ 1- University of Jyväskylä - Department of Physics, Nanoscience Center \\ FI-40014 Jyväskylä, Finland \\ 2- University of Jyväskylä - Faculty of Information Technology \\ FI-40014 Jyväskylä, Finland \\ 3- University of Jyväskylä - Department of Chemistry, Nanoscience Center \\ FI-40014 Jyväskylä, Finland
}

\begin{abstract}
Machine learning (ML) force fields are one of the most common applications of ML in nanoscience. However, commonly these methods are trained on potential energies of atomic systems and force vectors are omitted. Here we present a ML framework, which tackles the greatest difficulty on using forces in ML: accurate prediction of force direction. We use the idea of Minimal Learning Machine to device a method which can adapt to the orientation of an atomic environment to estimate the directions of force vectors. The method was tested with linear alkane molecules.
\end{abstract}

\section{Introduction}

In computational studies of atomic and molecular systems there are two fundamental quantities: potential energy of the system and force vectors subjecting to the atoms. In general, atomistic simulations produce output for both of these quantities. The most accurate way to compute them is to use ab initio methods, which are directly based on quantum mechanics. However, they are computationally demanding, which has risen the popularity of machine learning (ML) tools. This is due to the ability of ML to imitate the results of the high-level theoretical methods with lowered computational cost. Especially popular tools are ML force fields, which estimate high-dimensional potential energy surfaces of atomic systems [1]. These energy surfaces can be differentiated to get forces but the training the methods focuses on potential energies and forces are omitted.

Training a ML method to predict forces, instead of potential energies, is not simple. Chemical environments of the atoms are often presented using socalled descriptors. They produce translation, rotation and permutation invariant representations of the environment according to the chemical composition and geometry of the system [2]. They make regression tasks more feasible than in

\footnotetext{
*This work was supported by Academy of Finland through the AIPSE research program with grant 315549 to H.H. and 315550 to T.K., through the Universities Profiling Actions with grant 311877 to T.K., and through H.H.'s Academy Professorship. Work was also supported by "Antti ja Jenny Wihurin rahasto" via personal funding to A.P.. Computations were done at the FCCI node in the University of Jyväskylä (persistent identifier: urn:nbn:fi:research-infras2016072533).
} 
the case of using the atomic coordinates. Descriptors are highly useful but they are not suitable for predicting a rotation variant output, such as force directions, without major adjustments. However, if the description is rotation variant, the model would require large amounts of data to cover the orientation space.

We tackle the challenges above by utilizing the Minimal Learning Machine (MLM) [3] framework to create an orientation adaptive method. The main input is still an invariant description of a chemical environment. Output half of the method adjusts the spacial orientation of the reference data, which enables it to estimate force directions without having to cover the orientation space. Here we focus on the directions of the forces. Predicting the norms of the forces is a normal regression task, which can be handled with conventional ML methods such as Ridge regression or artificial neural networks.

\section{Theoretical basis of orientation adaptive MLM}

The general idea of the method is similar to the original MLM, which relies on separate handling of input and output spaces[3]. The force direction prediction splits into three parts, which use the descriptions of the chemical environments, coordinates of the atoms and unit force vectors. First, reference atomic coordinates are fitted on top of input coordinates, rotating reference unit force vectors respectively. Next, Euclidean distances are measured between input and reference descriptions forming a distance matrix, which is used to predict the cosines of angles between rotated reference force vectors and a force vector to be predicted. Finally, by minimizing the difference between real and predicted angles, the direction of the force is found.

\subsection{Training orientation adaptive MLM}

Three types of data are used in training: described chemical environments $\mathbf{X}=\left\{\mathbf{x}_{i}\right\}_{i=1}^{N} \in \mathbb{R}^{N \times d_{x}}$, cartesian coordinates of the atoms itselves and their $M$ nearest neighbors $\mathbf{Y}=\left\{\mathbf{y}_{i}\right\}_{i=1}^{N} \in \mathbb{R}^{N \times(1+M) \times 3}$, and unit vectors pointing to the directions of the forces $\mathbf{V}=\left\{\hat{\mathbf{v}}_{i}\right\}_{i=1}^{N} \in \mathbb{R}^{N \times 3}$. From this data $K$ references are selected forming $\mathbf{Q}=\left\{\mathbf{q}_{j}\right\}_{j=1}^{K} \in \mathbb{R}^{K \times d_{x}}, \mathbf{S}=\left\{\mathbf{s}_{j}\right\}_{j=1}^{K} \in \mathbb{R}^{K \times(1+M) \times 3}$ and $\mathbf{T}=\left\{\mathbf{t}_{j}\right\}_{j=1}^{K} \in \mathbb{R}^{K \times 3}$ respectively. In $\mathbf{y}_{i}$ and $\mathbf{s}_{i}$ the first rows are the positions of the analyzed atoms itselves.

The angle between force vectors can be measured reliably only if associated atomic neighborhoods are in the same spatial orientation, therefore reference neighborhoods in $\mathbf{S}$ are aligned with the ones in $\mathbf{Y}$. We used fitting method introduced by Arun et al.[4]. From point sets one calculates

$$
\mathbf{H}_{i, j}=\sum_{k=1}^{1+M}\left(\mathbf{y}_{i, k}-\mathbf{y}_{i, 1}\right)^{T}\left(\mathbf{s}_{j, k}-\mathbf{s}_{j, 1}\right)
$$

where $\mathbf{H}_{i, j} \in \mathbb{R}^{3 \times 3}$. Using Singular Value Decomposition (SVD) $\mathbf{H}_{i, j}=\mathbf{U} \boldsymbol{\Lambda} \mathbf{W}^{T}$ one can form a rotation matrix $\mathbf{R}_{i, j}=\mathbf{W} \mathbf{U}^{T}$. This aligns neighbor-atoms, when the analyzed atom itself is translated to the origin. The optimal order of $M$ 
neighbor-atoms is not known, therefore permutations are tested and the success of fitting is estimated as $g_{i, j}=\frac{1}{1+M} \sum_{k=1}^{1+M}\left|\left(\mathbf{y}_{i, k}-\mathbf{y}_{i, 1}\right)-\left(\mathbf{s}_{j, k}-\mathbf{s}_{j, 1}\right) \mathbf{R}_{i, j}\right|$. The permutation yielding the smallest $g_{i, j}$ is selected.

After SVD fitting, the matrices needed to train the model are formed. The basic training of the weight matrix $\mathbf{B} \in \mathbb{R}^{K \times K}$ is written as

$$
\mathbf{B}=\left(\mathbf{D}_{\text {in }}^{T} \mathbf{D}_{\text {in }}\right)^{-1} \mathbf{D}_{\text {in }}^{T} \mathbf{D}_{\text {out }}
$$

where $\mathbf{D}_{*} \in \mathbb{R}^{N \times K}$ are originally distance matrices in input and output spaces [3]. In our case there are three different $\mathbf{D}_{*}$ matrices: $\mathbf{D}_{x}=\left\{\left|\mathbf{x}_{i}-\mathbf{q}_{j}\right|\right\}$ containing Euclidean distances between chemical descriptions, $\mathbf{D}_{g}=\left\{\frac{1}{1+M} \sum_{k=1}^{1+M} \mid\left(\mathbf{y}_{i, k}-\right.\right.$ $\left.\left.\mathbf{y}_{i, 1}\right)-\left(\mathbf{s}_{j, k}-\mathbf{s}_{j, 1}\right) \mathbf{R}_{i, j} \mid\right\}$ with the goodness values of the SVD fittings and $\mathbf{D}_{c}=$ $\left\{\hat{\mathbf{v}}_{i} \cdot\left(\hat{\mathbf{t}}_{j} \mathbf{R}_{i, j}\right)\right\}$ having the cosines of the angles between force vectors in $\mathbf{V}$ and the rotated vectors of $\mathbf{T}$. We use equation (2) to train two weight matrices, $\mathbf{B}_{g}$ from goodness values of fittings and $\mathbf{B}_{c}$ from cosines. In both training processes $\mathbf{D}_{i n}=\mathbf{D}_{x}$ but the output side $\mathbf{D}_{\text {out }}$ matrix is substituted with $\mathbf{D}_{c}$ or $\mathbf{D}_{g}$ respectively. The purpose of two weight matrices is to use one to predict angles and another is used to determine reliability of the data points.

SVD fitting causes variation to $\mathbf{D}_{c}$ and $\mathbf{D}_{g}$ matrices, because configurations might be difficult to fit together. This variation is behaving as a semi-random noise, distribution of which is unclear. Hence, we used Huber regression to make the model robust to outliers [5]. The idea is similar to the robust MLM by Gomes et al. [6]. The columns of matrices $\mathbf{B}_{c}$ and $\mathbf{B}_{g}$ are optimized by giving $\mathbf{D}_{x}$ and columns of $\mathbf{D}_{c}$ or $\mathbf{D}_{g}$ to Huber regressor. The regressor also produces intercept values $c_{j}$ for every column of $\mathbf{B}$ to ensure that data is centered to origin. The robustness of the method is determined by the Huber parameter $\epsilon \in[1,2]$, where 1 is producing statistically the most robust model.

\subsection{Prediction of output direction}

The prediction takes a description $\mathbf{x}$ of the chemical environment and $\mathbf{y}$ set of coordinates of neighbor-atoms as an input. Euclidean distances between $\mathbf{x}$ and reference descriptions in $\mathbf{Q}$ are measured forming distance vector $\mathbf{d}_{x}$, which is used to estimate cosines and SVD fitting successes. With normally trained model this is simply $\mathbf{d}_{*}=\mathbf{d}_{x} \mathbf{B}_{*}$ and with robust trained model it is $\mathbf{d}_{*}=\mathbf{d}_{x} \mathbf{B}_{*}+\mathbf{c}_{*}$, where $\mathbf{c}_{*}$ contains intercept values. The reference points sets in $\mathbf{S}$ are SVD fitted to $\mathbf{y}$ producing success values of fittings, which are saved to vector $\mathbf{g}$, and rotation matrices to operate reference vectors in $\mathbf{T}$.

Only references for which $g_{j}-d_{g, j} \leq 0$ are used to predict the direction. Otherwise accuracy of the SVD fitting is not enough. Unit vector $\hat{\mathbf{u}}$ pointing to the predicted direction is found by minimizing loss function

$$
\min _{\hat{\mathbf{u}} \in \mathbb{R}^{3}} J(\hat{\mathbf{u}})=-\sum_{k \in \Gamma} \exp \left(-\left(\frac{d_{c, k}-\left(\hat{\mathbf{t}}_{k} \mathbf{R}_{k}\right) \cdot \hat{\mathbf{u}}}{\sigma_{1}}\right)^{2}-\left(\frac{g_{k}}{\sigma_{2}}\right)^{2}\right) .
$$

Here $\sigma_{1}$ and $\sigma_{2}$ are parameters defining the width and the depth of the contributions of the included references. $\Gamma$ contains the indeces of the accepted references. 
The optimization is done via Sequential Quadratic Programming (SQP). Initial guess is always a vector pointing from the atom itself to its nearest neighbor.

\section{$3 \quad$ Testing with alkanes}

Linear alkane molecules with number of carbon atoms ranging from two to seven were used as test systems. Thermal vibrations of the molecules were simulated by running molecular dynamics (MD) using Density Functional Tight-Binding (DFTB) code Hotbit to compute potential energies and forces [7]. For every atom initial velocities were generated from Maxwell-Boltzmann distribution with temperature of $750 \mathrm{~K}$. A single run was $1000 \mathrm{MD}$ steps $(1 \mathrm{step}=1$ configuration of the molecule) with 1.5 fs time step. Chemical environments were described using the Smooth Overlap of Atomic Positions (SOAP) [8] implemented in DScribe package [2]. SOAP parameters were set to $n_{\max }=6, l_{\max }=1$ and cut-off radius was $3.0 \AA$ (for further details see corresponding references). For the SVD fitting four nearest neighbors were used. The dataset was produced by two separate MD runs from all molecules $(6 \times 2 \times 1000$ configurations, $2 \times 27000$ carbon environments, $2 \times 66000$ hydrogen environments). Features in SOAP descriptions were min-max scaled into $[0,1]$ and training data of 7500 points was sampled for both elements using RS-maximin $[9,10]$. All training data points were saved as reference points. The descriptions of the hydrogen and carbon atoms were sampled separately and separate models were trained for both elements. For both elements one regular and eleven robust models were trained. For robust models Huber parameters were sampled evenly from the range $[1,2]$ with steps of 0.1. The third set of MD runs was used as test data. For carbon models, test data contained all data points from the third MD runs. For hydrogen, data points from the every second configurations of the molecules were used. The parameters in loss function (3) were $\sigma_{1}=0.25$ and $\sigma_{2}=0.5$. The performance was measured with weighted averages of the angles between predicted and real force vectors. The squared norms of the real force vectors were used as weights.

In Figure $1 \mathrm{~A}$ the weighted average angles are shown for different models. Training errors of regular models are out of visualization range. For hydrogen this training error is $1.3^{\circ}$ and for carbon $7.4^{\circ}$. Horizontal lines, representing the test errors of regular models, lie at $55.4^{\circ}$ for carbon and $42.1^{\circ}$ for hydrogen. Adding robustness increases training errors but generality is improved. For carbon all robust models are working better than the regular model, the best one producing the weighted average angle of $47.2^{\circ}$ with Huber parameter of 1.1. For hydrogen the effect of robustness is not as clear as for carbon. Only three most robust models show improvement and the best result with Huber parameter 1.0 is giving the weighted average angle of $38.3^{\circ}$. Panels D-G in Figure 1 show the test results for the regular models and the best robust models. In the case of carbon the effect of robustness is not clear. The main improvements lie in the region of large forces. For corresponding results for hydrogen effect is evident. The directions of large forces have improved. The directions of the small forces are difficult to handle, because even the tiniest movement of the atoms might 

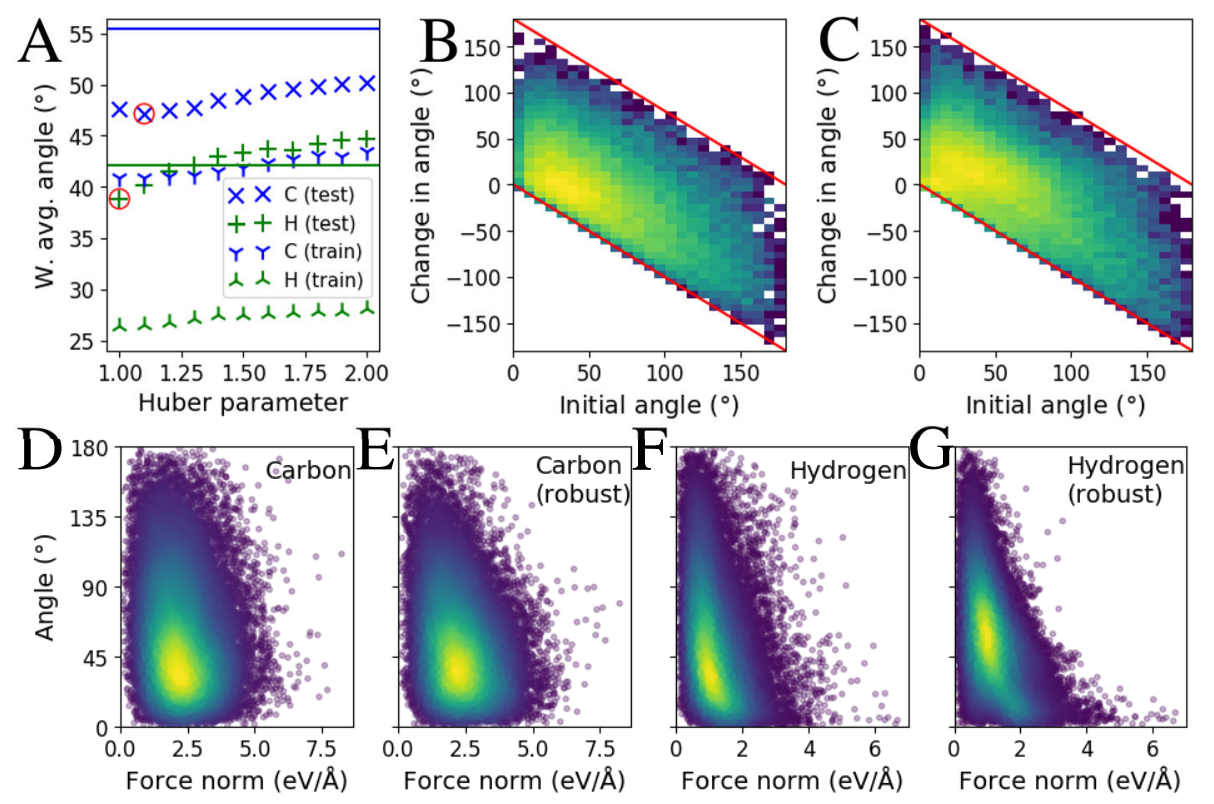

Fig. 1: Performance of the method. Panel A shows the weighted average angles between predicted and real directions. Lines show the test error of the regular models and crosses correspond to the robust ones with the best test results circled. Training errors of the regular models are below the visualization range. $\mathrm{B}$ and $\mathrm{C}$ show the effect of robustness from the best models of carbon and hydrogen respectively as 2D histograms. Colors are logarithmically normalized. Panels D-G show the test results. Colors present the density of the points.

totally change it. It is significantly more important to get correct directions for the large forces than for small ones. For hydrogen the robust model is starting to perform less well for small forces, which is seen as a different position of the density maximum.

In Fig. $1 \mathrm{~B}$ and $\mathrm{C}$ the effect of added robustness is visualized. Horizontal axes are the angles from the predictions with regular models. Vertical axes show the difference $\phi_{j}-\theta_{j}$, where $j \in\left[1, N_{\text {test }}\right], \theta_{j}$ is a angle produced by the regular model and $\phi_{j}$ is a corresponding angle from the best robust model. Negative values correspond to improved predictions. Lower side red line shows the optimal correction. Data is focusing to the lower region showing that robustness is mostly improving predictions. For carbon in panel B this is clear, because data is distributed close to the lower red line. For hydrogen improvement is modest. The maximum region is spread significantly along horizontal direction (no effect) and for small initial angles prediction have worsened but the main trend is improving. A similar behavior can be seen in Fig $1 \mathrm{~F}$ and G. 
ESANN 2021 proceedings, European Symposium on Artificial Neural Networks, Computational Intelligence and Machine Learning. Online event, 6-8 October 2021, i6doc.com publ., ISBN 978287587082-7.

Available from http://www.i6doc.com/en/.

\section{Conclusions}

Orientation adaptive MLM shows great promise on force direction prediction. Its advantage is that it is not bound to full atomic structures but local chemical environments are enough. The shown accuracy is not perfect but it could be improved by optimizing its several parameters such as the ones of the SOAP descriptor, the loss function and the number of reference points. We are also working to improve the fitting of atomic neighborhoods. A beautiful aspect of the method is that even after training the model, there are possibilities to affect its accuracy by tailoring the loss function and the optimization method. The future applications of the method lie in atomic structure optimization and MD simulations. However, direction estimation is not only important in nanoscience but also in, for example, engineering wind power[11] and predicting stock market[12]. Our method adds a new adjustable tool to tackle directional tasks.

\section{References}

[1] A. Pihlajamäki, J. Hämäläinen, J. Linja, et al. Monte Carlo Simulations of $\mathrm{Au}_{38}\left(\mathrm{SCH}_{3}\right)_{24}$ Nanocluster Using Distance-Based Machine Learning Methods. In J. Phys. Chem. A, vol. 124 p. 4827-4836, 2020. doi:10.1021/acs.jpca.0c01512.

[2] L. Himanen, M. O. J. Jäger, E. V. Morooka, et al. DScribe: Library of descriptors for machine learning in materials science. In Comput. Phys. Commun., vol. 247 p. 106949, 2020. doi:10.1016/j.cpc.2019.106949.

[3] A. H. de Souza Júnior, F. Corona, G. A. Barreto, et al. Minimal Learning Machine: A novel supervised distance-based approach for regression and classification. In Neurocomputing, vol. 164 pp. $34-44$, 2015. doi:10.1016/j.neucom.2014.11.073.

[4] K. S. Arun, T. S. Huang, and S. D. Blostein. Least-Squares Fitting of Two 3-D Point Sets. In IEEE T. Pattern Anal., vol. PAMI-9 pp. 698 - 700, 1987. doi: 10.1109/TPAMI.1987.4767965.

[5] P. J. Huber. Robust Statistics. John Wiley \& Sons, Inc, New Jersey, USA, 1981. ISBN 0-47141805-6.

[6] J. P. P. Gomes, D. P. P. Mesquita, A. L. Freire, et al. A Robust Minimal Learning Machine based on the M-Estimator. In ESANN 2017 proceedings, European Symposium on Artificial Neural Networks, Computational Intelligence and Machine Learning, pp. 383-388. 2017.

[7] P. Koskinen and V. Mäkinen. Density-functional tight-binding for beginners. In Comp. Mater. Sci., vol. 47(1) pp. 237 - 253, 2009. ISSN 0927-0256. doi: 10.1016/j.commatsci.2009.07.013.

[8] A. P. Bartók, R. Kondor, and G. Csányi. On representing chemical environments. In Phys. Rev. B, vol. 87, 2013. doi:10.1103/PhysRevB.87.184115.

[9] T. F. Gonzalez. Clustering to minimize the maximum intercluster distance. In Theor. Comput. Sci., vol. 38 pp. 293-306, 1985. doi:10.1016/0304-3975(85)90224-5.

[10] J. Hämäläinen, A. S. C. Alencar, T. Kärkkäinen, et al. Minimal Learning Machine: Theoretical Results and Clustering-Based Reference Point Selection. In J. Mach. Learn. Res., vol. 21(239) pp. 1-29, 2020. URL http://jmlr.org/papers/v21/19-786.html.

[11] Z. Tang, G. Zhao, and T. Ouyang. Two-phase deep learning model for short-term wind direction forecasting. In Renew. Energ., vol. 173 pp. 1005-1016, 2021. doi: 10.1016/j.renene.2021.04.041.

[12] M. Ballings, D. V. den Poel, N. Hespeels, et al. Evaluating multiple classifiers for stock price direction prediction. In Expert Syst. Appl., vol. 42 pp. 7046-7056, 2015. doi: 10.1016/j.renene.2021.04.041. 